Correspondence: Thomas Eiwegger, Dept of Pediatrics and Adolescent Medicine, Medical University of Vienna, Waehringer Guertel 18-20, 1090 Vienna, Austria. E-mail: thomas.eiwegger@meduniwien.ac.at

Received: March 102014 | Accepted after revision: April 142014 | First published online: June 252014

Support statement: Supported by funds from the Oesterreichische Nationalbank (Anniversary Fund, project number $13846 \mathrm{ONB}$ ) and the Medical Scientific Fund of the Mayor of the City of Vienna (number 11013).

Conflict of interest: Disclosures can be found alongside the online version of this article at erj.ersjournals.com

\title{
References
}

1 Tiringer K, Treis A, Fucik P, et al. A Th17- and Th2-skewed cytokine profile in cystic fibrosis lungs represents a potential risk factor for Pseudomonas aeruginosa infection. Am J Respir Crit Care Med 2013; 187: 621-629.

2 Liou TG, Adler FR, Keogh RH, et al. Sputum biomarkers and the prediction of clinical outcomes in patients with cystic fibrosis. PLoS One 2012; 7: e42748.

3 Hirsiger S, Simmen HP, Werner CM, et al. Danger signals activating the immune response after trauma. Mediators Inflamm 2012; 2012: 315941.

4 Akdis M, Burgler S, Crameri R, et al. Interleukins, from 1 to 37, and interferon- $\gamma$ : receptors, functions, and roles in diseases. J Allergy Clin Immunol 2011; 127: 701-721.e70.

5 Roussel L, Farias R, Rousseau S. IL-33 is expressed in epithelia from patients with cystic fibrosis and potentiates neutrophil recruitment. J Allergy Clin Immunol 2013; 131: 913-916.

6 Schmitz J, Owyang A, Oldham E, et al. IL-33, an interleukin-1-like cytokine that signals via the IL-1 receptorrelated protein ST2 and induces T helper type 2-associated cytokines. Immunity 2005; 23: 479-490.

7 Yanai H, Ban T, Taniguchi T. High-mobility group box family of proteins: ligand and sensor for innate immunity. Trends Immunol 2012; 33: 633-640.

8 Abraham E, Arcaroli J, Carmody A, et al. HMG-1 as a mediator of acute lung inflammation. J Immunol 2000; 165: 2950-2954.

9 Rowe SM, Jackson PL, Liu G, et al. Potential role of high-mobility group box 1 in cystic fibrosis airway disease. Am J Respir Crit Care Med 2008; 178: 822-831.

10 Wang $\mathrm{H}$, Liao $\mathrm{H}$, Ochani $\mathrm{M}$, et al. Cholinergic agonists inhibit HMGB1 release and improve survival in experimental sepsis. Nat Med 2004; 10: 1216-1221.

11 Brazova J, Sediva A, Pospisilova D, et al. Differential cytokine profile in children with cystic fibrosis. Clin Immunol 2005; 115: 210-215.

12 Hartl D, Griese M, Kappler M, et al. Pulmonary Th2 response in Pseudomonas aeruginosa-infected patients with cystic fibrosis. J Allergy Clin Immunol 2006; 117: 204-211.

\section{Interferon- $\gamma$ responses to Mycobacterium tuberculosis-specific antigens in diabetes mellitus}

To the Editor:

Although diabetes mellitus has long been recognised as a risk factor for tuberculosis, it was only recently that strong evidence for this emerged [1]. Persons with diabetes mellitus have a two or three times higher risk of developing tuberculosis disease than nondiabetics; those with tuberculosis and diabetes mellitus have a four times higher risk of death during tuberculosis treatment and a higher risk of tuberculosis relapse [2, 3].

Diabetics therefore constitute a target group in whom the identification of latent tuberculosis infection (LTBI) and its treatment may potentially be an important strategy for tuberculosis elimination $[4,5]$. Interferon- $\gamma$ release assays (IGRAs) are immunodiagnostic tests for identification of LTBI. These tests have shown superior specificity and positive predictive value for progression to active disease over the tuberculin skin test [6-9]. Although the IGRAs do not distinguish active from latent tuberculosis [10, 11], they are often done as part of the work-up for active tuberculosis in cases where diagnostic uncertainty exists. To date, there is scant information in the literature regarding the performance of these assays in diabetics.

In a previous report in which we evaluated the T-SPOT.TB (Oxford Immunotec, Abingdon, UK) and QuantiFERON In-Tube (QFT-IT) (Cellestis, Melbourne, Australia) assays in a head-to-head manner in 270 culture-confirmed pulmonary tuberculosis patients, we had found undiminished sensitivity of these assays in the presence of diabetes mellitus [12]. WALSH et al. [13] have also reported that diabetes did not affect the performance of the second-generation QuantiFERON TB Gold (QFT-G) and T-SPOT.TB [13]. 
In the present study, we compared the quantitative T-cell responses to Mycobacterium tuberculosis-specific antigens as measured by the T-SPOT.TB and QFT-IT, and to mitogen as measured by the QFT-IT, among diabetics and nondiabetics with culture-confirmed pulmonary tuberculosis. We also evaluated these responses according to diabetic control as indicated by glycated haemoglobin (HbAlc) levels.

Data for this analysis were extracted from a main study designed to evaluate the effect of tuberculosis treatment on the T-SPOT.TB and QFT-IT in a head-to-head manner [11]. This study was approved by the Domain Specific Institutional Review Board of the National Healthcare Group (Singapore). The study population comprised pulmonary tuberculosis patients treated at the Singapore Tuberculosis Control Unit who were prospectively recruited between April 2006 and February 2007 within 2 weeks of starting tuberculosis treatment. All study participants gave informed consent. At least two sputum specimens were obtained on separate days for acid-fast bacilli (AFB) smears and tuberculosis culture and drug sensitivity testing prior to starting treatment. Peripheral venous blood was drawn for both IGRAs at the time of recruitment. HIV testing was routinely offered. Baseline liver enzymes, serum creatinine and random blood glucose level were routinely performed. For this study, patients were classified as diabetic if they had history of diabetes at the time of tuberculosis diagnosis, or if they were found to have two random glucose level measurements $>11.0 \mathrm{mmol} \cdot \mathrm{L}^{-1}$ at baseline and on repeat testing. Patients with guarded prognoses (e.g. the frail elderly or those with co-existing advanced malignancy), HIV and those who could not be followed-up for relapse (e.g. non-Singapore residents) were excluded from the study. Data on patient demographics, comorbidities, bacteriological status and radiological findings were captured.

The T-SPOT.TB and QFT-IT assays were performed according to the manufacturers' instructions at the Tan Tock Seng Hospital microbiology laboratory. The T-SPOT.TB was considered reactive if either or both of panel A (containing the $6-\mathrm{kDa}$ early secretory antigen target (ESAT-6)) or panel B (containing the $10-\mathrm{kDa}$ culture filtrate protein $(\mathrm{CFP}-10))$ had $\geqslant 6$ more spots than the negative control and this number was at least twice the number of spots in the negative control. The QFT-IT was considered positive if the interferon (IFN)- $\gamma$ measured in the antigen tube was $\geqslant 0.35 \mathrm{IU} \cdot \mathrm{mL}^{-1}$ above that produced in the negative control tube. Indeterminate results were excluded from the multivariate analysis.

Analysis was performed using SPSS for Windows version 17 (IBM, Armonk, NY, USA). The statistics for qualitative data were performed using the Chi-squared test or Fisher's exact test while the quantitative data were analysed by Mann-Whitney U-test and Kruskal-Wallis test. Multivariate models were used to test the association between diabetes and IGRA positivity rate (logistic regression) or quantitative responses (linear regressions), by adjusting for potential confounding factors (age, sex, ethnicity, cavitary disease, body mass index (BMI), smoking and smear positivity). A p-value $<0.05$ was regarded as statistically significant.

There were 99 diabetic and 176 nondiabetic patients. Their baseline characteristics, IGRA positivity rates and quantitative results (mean spot-forming cells and IFN- $\gamma$ concentrations) are shown in table 1. Diabetics were more likely to be older (mean age 54 years), of non-Chinese (i.e. Malay or Indian) ethnicity, to have cavitary disease, have a BMI $\geqslant 19 \mathrm{~kg} \cdot \mathrm{m}^{-2}$ and to be sputum AFB smear-positive. By univariate analysis, there were no statistically significant differences in the IGRA positivity rates and the quantitative responses between diabetic and nondiabetic tuberculosis patients. However, the QFT-IT indeterminate rate was significantly higher in diabetic than nondiabetic tuberculosis patients $(7.1 \%$ versus $1.7 \%, \mathrm{p}=0.039)$.

Multivariate logistic regression analysis showed that, after taking into account the concurrent influences of age, sex, ethnicity, BMI, smoking, smear status and chest-radiographic cavitation, the IGRAs performed as well in tuberculosis patients with diabetes as in those without diabetes (T-SPOT.TB, $\mathrm{p}=0.108$; QFT-IT, $\mathrm{p}=0.831$ ). Moreover, in linear regression analysis, there was no significant difference in the response to ESAT-6 $(\mathrm{p}=0.538)$ and CFP-10 $(\mathrm{p}=0.892)$ or antigen-nil $(\mathrm{p}=0.084)$ and mitogen-nil $(\mathrm{p}=0.702)$ between diabetic and nondiabetic tuberculosis patients. $77(78 \%)$ of our diabetic patients had HbAlc levels measured at baseline. Of these, 10 (12.9\%), $36(46.8 \%)$ and $31(40.3 \%)$ had HbAlc <7\%, 7-10\% and $>10 \%$, respectively. The median HbAlc value was 9.5\% (range 4.9-15\%). By univariate analysis, there was no significant difference in the AFB smear positivity $(\mathrm{p}=0.226)$, cavitary status $(\mathrm{p}=0.659)$ and quantitative responses (median) of both IGRAs: ESAT-6 $(\mathrm{p}=0.495)$, CFP-10 $(\mathrm{p}=0.341)$, antigen-nil $(\mathrm{p}=0.774)$ and mitogen-nil $(\mathrm{p}=0.841)$ among those with diabetes and $\mathrm{HbA1c}<7 \%, 7-10 \%$ and $>10 \%$, respectively.

We found no significant differences in the qualitative or quantitative T-cell IFN- $\gamma$ responses to M. tuberculosis-specific antigens as measured by the QFT-IT and T-SPOT.TB assays between diabetic and nondiabetic patients with culture-confirmed pulmonary TB. There was also no significant difference in the quantitative responses to mitogen as measured by the QFT-IT between diabetics and nondiabetics. The performance of both IGRAs was unaffected by diabetes mellitus control as reflected by HbAlc levels at the time of tuberculosis diagnosis. 
TABLE 1 Comparison of characteristics and interferon- $\gamma$ release assay (IGRA) results between diabetic and nondiabetic subjects, and multivariate analysis models for IGRA responses (positivity rate and quantitative) among diabetic patients

\begin{tabular}{|c|c|c|c|c|c|}
\hline Comparison of characteristics & \multicolumn{2}{|c|}{ Diabetics } & Nondiabetics & \multicolumn{2}{|c|}{ p-value ${ }^{\#}$} \\
\hline Subjects $n$ & \multicolumn{2}{|c|}{99} & 176 & \\
\hline Age years mean & \multicolumn{2}{|c|}{54.3} & 43.2 & \multicolumn{2}{|l|}{0.001} \\
\hline Males & \multicolumn{2}{|c|}{$77(77.8)$} & $126(71.6)$ & \multicolumn{2}{|l|}{0.263} \\
\hline Non-Chinese ethnicity & \multicolumn{2}{|c|}{$43(43.4)$} & $46(26.1)$ & \multicolumn{2}{|l|}{0.003} \\
\hline Cavitary lesion on chest radiography & \multicolumn{2}{|c|}{$64(64.6)$} & $76(43.2)$ & \multicolumn{2}{|l|}{0.001} \\
\hline Sputum AFB smear positive & \multicolumn{2}{|c|}{86 (86.9) } & $124(70.5)$ & \multicolumn{2}{|l|}{0.002} \\
\hline $\mathrm{BMI} \geqslant 19 \mathrm{~kg} \cdot \mathrm{m}^{-2}$ & \multicolumn{2}{|c|}{$76(77.6)$} & $72(41.1)$ & \multicolumn{2}{|c|}{$<0.001$} \\
\hline Ever-smokers & \multicolumn{2}{|c|}{$58(58.6)$} & $95(54.0)$ & \multicolumn{2}{|l|}{0.460} \\
\hline \multicolumn{6}{|l|}{ T-SPOT.TB assay } \\
\hline Positive & \multicolumn{2}{|c|}{92 (92.9) } & $162(92.0)$ & \multicolumn{2}{|l|}{0.791} \\
\hline Failure & \multicolumn{2}{|c|}{$2(2.0)$} & $2(1.1)$ & \multicolumn{2}{|l|}{0.621} \\
\hline Response to ESAT-6 SFCs per 250000 PBMCs median & \multicolumn{2}{|c|}{28} & 25 & \multicolumn{2}{|l|}{0.717} \\
\hline Response to CFP-10 SFCs per 250000 PBMCs median & & & 37 & 0.773 & \\
\hline QFT-IT assay & & & & & \\
\hline Positive & 74 & & $147(83.5)$ & 0.079 & \\
\hline Indeterminate & & & $3(1.7)$ & 0.039 & \\
\hline Antigen-nil response IU $\cdot \mathrm{mL}^{-1}$ median & & & 2.0 & 0.474 & \\
\hline Mitogen-nil response IU $\cdot \mathrm{mL}^{-1}$ median & & & 13.6 & 0.665 & \\
\hline Positivity rate & Logistic $r$ & ession & Quantitative response & Linear regre & ssion \\
\hline & $\begin{array}{c}\text { Adjusted } \\
\text { OR }(95 \% \mathrm{Cl})\end{array}$ & $\begin{array}{c}\text { Adjusted } \\
\text { p-value }\end{array}$ & & $\begin{array}{c}\text { Adjusted } \beta \\
\text { coefficient } \\
(95 \% \mathrm{Cl})\end{array}$ & $\begin{array}{c}\text { Adjusted } \\
\text { p-value }\end{array}$ \\
\hline T-SPOT.TB positivity & 3.02 & 0.108 & ESAT-6 & $1.14(0.75-1.72)$ & 0.538 \\
\hline rate & $(0.78-11.64)$ & & CFP-10 & $1.03(0.64-1.65)$ & 0.892 \\
\hline QFT-IT positivity & 1.09 & 0.831 & Antigen-nil & $1.30(0.96-1.76)$ & 0.084 \\
\hline rate & $(0.48-2.49)$ & & Mitogen-nil & $0.53(0.02-13.75)$ & 0.702 \\
\hline
\end{tabular}

Data are presented as $\mathrm{n}(\%)$, unless otherwise stated. AFB: acid-fast bacilli; BMI: body mass index; ESAT-6: 6-kDa early secretory antigenic target; SFC: spot-forming cell; PBMC: peripheral blood mononuclear cell; CFP-10: 10-kDa culture filtrate protein; QFT-IT: QuantiFERON In-Tube. \#: Chi-squared, Fisher's exact or unpaired t-test.

WALSH et al. [13] found a significantly increased sensitivity of the QFT-G and higher secretion of IFN- $\gamma$ in response to QFT-G antigens in diabetics than in nondiabetic tuberculosis patients. They attributed this to the observation that diabetic patients have higher bacterial burden, resulting in more robust stimulation of IFN- $\gamma$. However, in the univariate analysis, we found a lower sensitivity $(74.7 \%$ versus $83.5 \%, \mathrm{p}=0.079)$ and a significantly higher indeterminate rate of the QFT-IT $(7.1 \%$ versus $1.7 \%, \mathrm{p}=0.039)$ in diabetics than in nondiabetics, despite our diabetics being significantly more likely to have smear-positive and cavitary disease. In contrast, the T-SPOT.TB assay showed a consistently low test failure rate ( $2.0 \%$ versus $1.1 \%)$, and high sensitivity ( $92.9 \%$ versus $92 \%$ ) in both diabetics and nondiabetics, a finding that is corroborated by WALSH et al. [13].

A limitation of our study is that newly diagnosed diabetes mellitus was based on two random glucose readings of $>11 \mathrm{mmol} \cdot \mathrm{L}^{-1}$, which may have resulted in overdiagnosis of diabetes, as acute infection may cause transient hyperglycaemia.

This study demonstrates that IFN- $\gamma$ production in diabetic tuberculosis patients is independent of microbiological burden. While it adds to current evidence that the sensitivity of the IGRAs is unaffected in diabetic patients with active tuberculosis, it should be noted that the performance of the QFT-IT falls short of that of the T-SPOT.TB in these patients. 
Suay-Hong Gan ${ }^{1}$, Kyi-Win KhinMar ${ }^{1}$, Timothy M. Barkham ${ }^{2}$, Chwee-Kim Koh $^{1}$, Liang Shen ${ }^{3}$, Yee-Tang Wang ${ }^{1}$ and Cynthia B.E. Chee ${ }^{1}$

${ }^{1}$ Tuberculosis Control Unit, Tan Tock Seng Hospital, Singapore. ${ }^{2}$ Dept of Pathology and Laboratory Medicine, Tan Tock Seng Hospital, Singapore. ${ }^{3}$ Biostatistics Unit, National University of Singapore, National University Health System, Singapore.

Correspondence: Suay-Hong Gan, Tuberculosis Control Unit, Tan Tock Seng Hospital, 144 Moulmein Road, 308089, Singapore. E-mail: suay_hong_gan@ttsh.com.sg

Received: Dec 262013 | Accepted after revision: April 022014 | First published online: April 172014

Support statement: This study was funded by the Singapore National Medical Research Council (grant 1004/2005).

Conflict of interest: Disclosures can be found alongside the online version of this article at erj.ersjournals.com

\section{References}

World Health Organization. Collaborative framework for care and control of tuberculosis and diabetes. WHO/ HTM/TB/2011.15. Geneva, WHO, 2011.

2 Jeon CY, Murray MB. Diabetes mellitus increases the risk of active tuberculosis: a systematic review of 13 observational studies. PLoS Med 2008; 5: e152.

3 Baker MA, Harries AD, Jeon CY, et al. The impact of diabetes on tuberculosis treatment outcomes: a systematic review. BMC Medicine 2011; 9: 81.

4 Diel R, Loddenkemper R, Zellweger JP, et al. Old ideas to innovate tuberculosis control: preventive treatment to achieve elimination. Eur Respir J 2013; 42: 785-801.

5 D'Ambrosio L, Dara M, Tadolini M, et al. Tuberculosis elimination: theory and practice in Europe. Eur Respir J 2014; 43: 1410-1420.

6 Diel R, Goletti D, Ferrara G, et al. Interferon- $\gamma$ release assays for the diagnosis of latent Mycobacterium tuberculosis infection: a systematic review and meta-analysis. Eur Respir J 2011; 37: 88-99.

7 Diel R, Loddenkemper R, Nienhaus A. Predictive value of interferon- $\gamma$ release assays and tuberculin skin testing for progression from latent TB infection to disease state: a meta-analysis. Chest 2012; 142: 63-75.

8 Migliori GB, Zellweger JP, Abubakar I, et al. European Union standards for tuberculosis care. Eur Respir J 2012; 39: $807-819$.

9 Migliori GB, Sotgiu G, Blasi F, et al. Towards the development of EU/EEA Standards for Tuberculosis Care (ESTC). Eur Respir J 2011; 38: 493-492.

10 Goletti D, Stefania C, Butera O, et al. Accuracy of immunodiagnostic tests for active tuberculosis using single and combined results: a multicentre TBNET-study. PloS One 2008; 3: e3417.

11 Jafari C, Thijsen S, Sotgiu G, et al. Bronchoalveolar lavage enzyme-linked immunospot for a rapid diagnosis of tuberculosis. Am J Respir Crit Care Med 2009; 180: 666-673.

12 Chee CBE, Gan SH, Khinmar KW, et al. Comparison of sensitivities of two commercial gamma interferon release assays for pulmonary tuberculosis. J Clin Microbiol 2008; 46: 1935-1940.

13 Walsh MC, Camerlin AJ, Miles R, et al. The sensitivity of interferon- $\gamma$ release assays is not compromised in tuberculosis patients with diabetes. Int J Tuberc Lung Dis 2011; 15: 179-184.

14 Kabeer BSA, Balambal R, Thomas A, et al. Role of QuantiFERON-TB Gold, interferon gamma inducible preotein10 and tuberculin skin in active tuberculosis diagnosis. PloS One 2010; 5: e9051.

\section{In vitro synergy between linezolid and clarithromycin against Mycobacterium tuberculosis}

To the Editor:

Approximately 3\% of new tuberculosis cases worldwide represent multidrug-resistant tuberculosis (MDRTB) [1]. In these MDR-TB cases, resistance of Mycobacterium tuberculosis to the otherwise effective rifampicin and isoniazid forces clinicians to diverge to other antimicrobial agents. Such treatment options include the World Health Organization (WHO) group 5 drugs linezolid and clarithromycin [1]. Linezolid shows excellent efficacy in the treatment of MDR-TB, but its use is often troubled by adverse events [2-4]. Linezolid has shown in vitro bacteriostatic activity against $M$. tuberculosis and is also effective at achieving culture conversion in drug-resistant cases [5]. In vitro testing revealed that clarithromycin is not very active against $M$. tuberculosis, as the minimal inhibitory concentrations (MICs) are relatively high. Clinical efficacy seems questionable, as MICs, as reported in the literature, are significantly higher than achievable serum peak levels in vivo [6]. Conversely, clarithromycin reaches adequate local concentrations in alveolar cells 